\title{
Jobs that Fulfil the Needs of Extraterrestrial Living
}

In the words of Cpt. Jean-Luc Picard, 'Things are only impossible until they are not'.

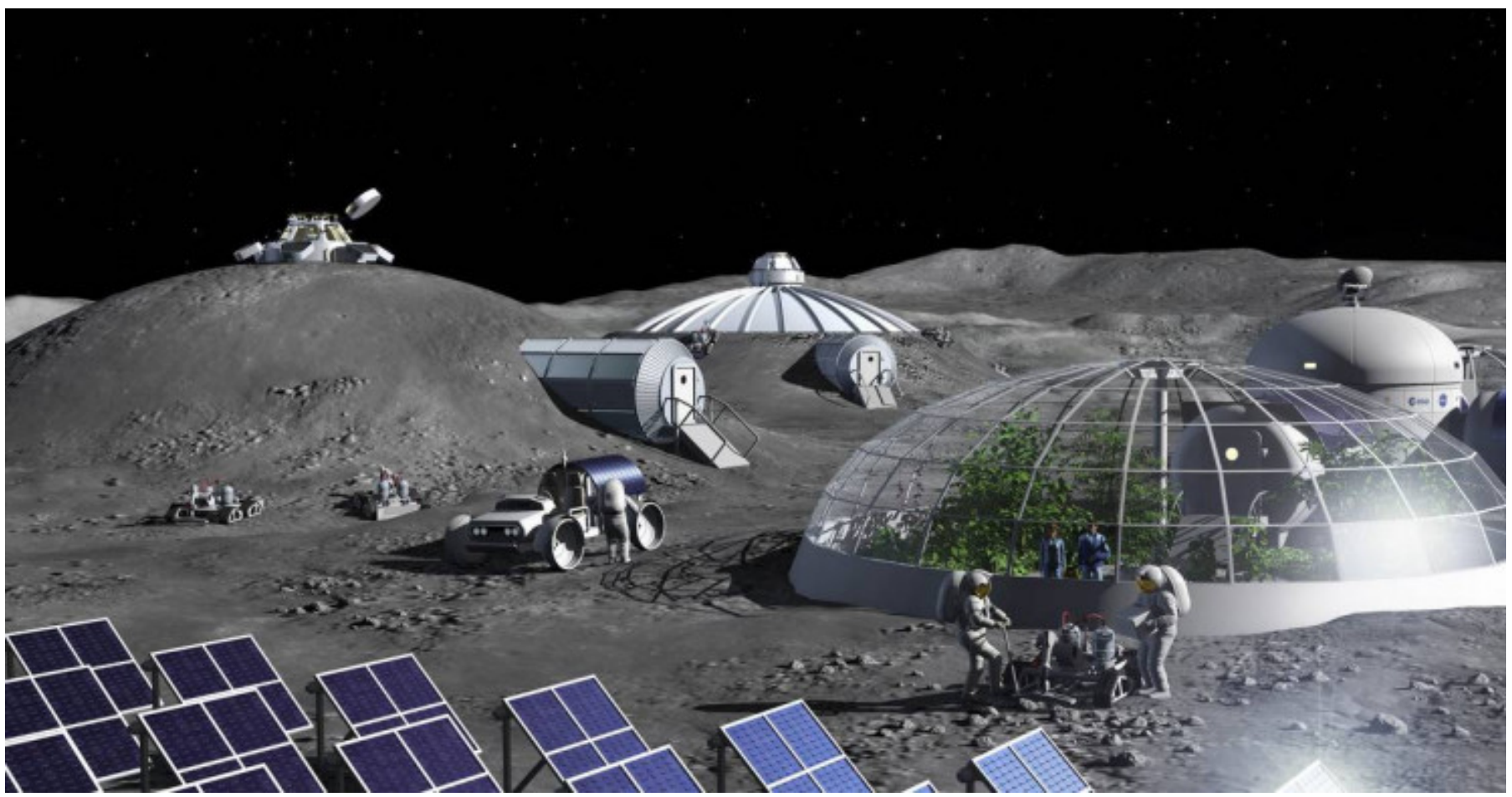

Artist impression of activities in a Moon Base. Credit: ESA -P. Carril

\section{0 words}

Only five years ago talk of space economies was viewed by many as the stuff of science fiction. Those like me with extraterrestrial interests - and moulded in part by Star Trek's cast boldly exploring the cosmos during our youth - have occasionally been chastised as dreamers. Yet, the realms of possibility are changing. Chemists have big roles to play as humankind blazes new trails - both on Earth and other worlds.

The UK space economy has more than trebled in size since 2000, supporting approximately 42,000 jobs. Headline initiatives such as the recently announced horizontal and vertical launch space ports for the UK, as well as Space Park Leicester, demonstrate heavy investment in the scientific, manufacturing and technological base linked with the rapidly growing global space economy. Alone, the space port projects will create thousands of new jobs, driving all manner of exciting new innovations with a range of efficiencyimproving applications on Earth. Yet more new employment opportunities shall arise from partnerships with government space agencies and private sector bodies, and connected with a lunar economy when the Lunar Gateway space outpost is established following its launch in 2024. The international space market is estimated to be worth $£ 400$ billion by 2030 and the UK is building toward a $10 \%$ share of this, which would account for upwards of 100,000 new jobs.

The planetary science research sector that employs a range of physical scientists, and includes the UK's specialist Cosmochemical Analytical Network, is also growing. A wave of excitement swept the globe when the Perseverance Rover touched down on Mars on 18 February to collect samples; thrills to be renewed when the rocks travel to Earth for analysis in 2031. Other samples retrieved from the Moon (by the Chang'e-5 mission) and asteroids (by Hyabusa2) landed on Earth in late 2020, and more are due in 2023 (OSIRIS-REX). Plus, despite the lockdown the people and skies of the UK lit up on 28 February when the first recovered meteorite for 30 years fell near Winchcombe. These shards of rock and soils from space, while providing critical insights into our Solar System's history, will guide us in the practical needs of off-Earth settlements and further space exploration. 
With accelerating growth in space and planetary sciences comes all manner of fresh prospects and challenges for chemists. Materials brought back from space will be curated in dedicated, bio-secure facilities. They will then be subject to extensive interrogations via specialist imaging and microscopy equipment, analytical chemistry involving gas chromatography, and wet-chemical processing to scrutinise organic and inorganic chemical systematics at rock to atomic scales; drawing on the latest and highprecision, high-sensitivity mass spectrometers.

Chemical knowledge will be vital for creating new forms of batteries and fuels to power rockets and extraterrestrial living. Bespoke material sorting and innovative synthetic chemical processing methods will be needed to harvest resources such as oxygen, energy, critical metals and construction materials from other worlds, mandating new processes efficient in the extreme. And when space settlements become a reality, chemists will also be vital in developing extraterrestrial habitats that will sustainably cycle and recycle water, oxygen, and nutrients indispensable to food production while simultaneously regulating temperatures and minimising waste. The design of these settlements, integrated with knowledge from human experiments in microgravity aboard the International Space Station, must support astronaut health and wellbeing while offering protection from the threats of high radiation and meteorite bombardment on celestial bodies where atmospheres and shielding magnetic fields are absent or weak. Those astronauts including the first woman to step on the Moon - could be chemists, as many past astronauts have been. The effects of space exploration will also be felt back here on Earth. Technological innovations driven by humankind's extraterrestrial endeavours have long helped to improve lives. The Apollo programme and International Space Station gave us cochlear implants, dustbuster vacuums, memory foam, effective insulation materials, improved water purification, and the culinary delights of freeze-dried food. Recently, the space sector has created many new green jobs within the UK, such as Solar Energy Research Engineers, with 15,000 more green roles forecast by 2030 and additional benefits and opportunities for Europe; all helping to progress sustainable practices and achieve net zero carbon.

To prepare for this future we should be inspired by Star Trek's rich mixes of talent among crews of the Starships Enterprise and Voyager. In this age of internationally cooperative space exploration, diverse teams and their ways of thinking are just and crucial to maximising scientific and innovation potential. As our time as custodians of Earth has been far from exemplary due to our fractious wars, environmental and ecological impacts, and persisting social and workplace inequalities, we have a collective duty to do significantly better in settling on new worlds.

The prospects for chemists are there. Now is the time to live these dreams.

By Amy J. V. Riches

Planetary Scientist, Geochemist, Author and Editor

Visiting Honorary Fellow, University of Edinburgh, UK

Affiliate Scientist, SETI Institute, USA.

Website: https://amyriches.org/

Twitter: @PlanetaryAmy 